\title{
CHARACTERISTIC FUNCTIONS AND AVERAGES
}

\author{
Assis Azevedo ${ }^{1}$ \\ Department of Mathematics and Applications, University of Minho \\ Campus de Gualtar, 4710-057 Braga, Portugal \\ assis@math.uminho.pt
}

\begin{abstract}
Let $\Omega$ be a set and $\Omega_{1}, \ldots, \Omega_{m-1}$ subsets of $\Omega$, being $m$ an integer greater than one. For a given function $f=\left(f_{1}, \ldots f_{m}\right): \Omega \rightarrow \mathbb{R}^{m}$, we prove the existence of a unique function $\alpha=\left(\alpha_{1}, \ldots, \alpha_{m}\right): \Omega \rightarrow \mathbb{R}^{m}$ such that$$
\left\{\begin{array}{l}
\alpha_{i} \\
\alpha_{1}+\cdots+\alpha_{i}=\alpha_{i+1} \text { on } \Omega_{i} \\
\alpha_{1}+\cdots+\alpha_{m}=f_{1}+\cdots+f_{m},
\end{array} \text { on } \Omega \backslash \Omega_{i}, \text { for all } i<m\right.
$$

called the average function of $f: \Omega \rightarrow \mathbb{R}^{m}$ relatively to $\left(\Omega, \Omega_{1}, \ldots, \Omega_{m-1}\right)$.

When $\Omega$ is a topological space and $f$ is a continuous function, we find necessary and sufficient conditions for the continuity of the average function of $f$.

We write $\alpha_{i}$ as a linear combination of characteristic functions of the (coincidence) sets $\cap_{j=r}^{s} \Omega_{j}, 1 \leq r \leq s \leq m-1$, belonging the coefficients to $\mathbb{Q}\left[f_{1}, \ldots, f_{m}\right]$.
\end{abstract}

\section{Introduction}

The motivation of this paper had its origin in a joint work with J. F. Rodrigues and L. Santos (see [1]). The problem under consideration there, was a variational inequality modelling the problem of equilibrium of $N$ membranes, attached to rigid supports on the boundary, each one under the action of given forces $\left(f_{1}, \ldots, f_{N}\right)$ and constrained by the other membranes (see Remark 3.2).

Let $\Omega$ be a set, $m>1$, an integer, $\Omega_{i}, 1 \leq i \leq m-1$ subsets of $\Omega$. It is easy to characterize the sets

$$
\begin{aligned}
& \mathcal{A}^{m}=\left\{\alpha \in \mathcal{F}(\Omega)^{m}: \Omega_{i} \subseteq\left\{x \in \Omega: \alpha_{i}(x)=\alpha_{i+1}(x)\right\}, \text { for } 1 \leq i \leq m-1\right\}, \\
& \mathcal{B}^{m}=\left\{\alpha \in \mathcal{F}(\Omega)^{m}: \Omega_{i}=\left\{x \in \Omega: \alpha_{i}(x)=\alpha_{i+1}(x)\right\}, \text { for } 1 \leq i \leq m-1\right\},
\end{aligned}
$$

where $\mathcal{F}(\Omega)$ is the set of real-valued functions with domain $\Omega$.

For instance, $\alpha=\left(\alpha_{1}, \ldots, \alpha_{n}\right) \in \mathcal{A}^{m}$ if and only if there exists $f_{1}, \ldots, f_{m}$ such that $\alpha_{1}=f_{1}$ and $\alpha_{i}=\alpha_{i-1}+f_{i} h_{i-1}$ where $h_{i}$ is a function that vanishes on $\Omega_{i}$, for example the characteristic function of $\Omega \backslash \Omega_{i}$ or, in the case we are in a

\footnotetext{
${ }^{1}$ Work supported by the Research Centre of Mathematics of the University of Minho through the FCT Pluriannual Funding Program.
} 
metric space, the distance function to $\Omega_{i}$. In both cases it is easy to know when $\alpha$ belongs to $\mathcal{B}^{m}$ and when it is continuous. But in none of these cases we have $\alpha_{1}+\cdots+\alpha_{m}=f_{1}+\cdots+f_{m}$.

The family $\left(\Omega_{i}\right)_{i=1, \ldots, m-1}$ allows us to define $m$ partitions of $\Omega$ that are crucial to the construction of the average functions. In what follows we consider $\Omega_{0}=\Omega_{m}=\emptyset$. We also construct partitions to $\Omega \backslash \Omega_{i}$, for $i=1, \ldots, m-1$.

Definition 1.1. For $1 \leq r \leq s \leq m$ let

$$
\Omega_{r, s}=\left(\bigcap_{r \leq k \leq s-1} \Omega_{k}\right) \backslash\left(\Omega_{r-1} \cup \Omega_{s}\right) \quad \text { if } 1 \leq r \leq s \leq m .
$$

Notice that, with the usual convention about intersections, $\Omega_{r, r}=\Omega \backslash\left(\Omega_{r-1} \cup \Omega_{r}\right)$.

Lemma 1.2. For each $i=1, \ldots, m$,

a) $\left(\Omega_{r, s}\right)_{1 \leq r \leq i \leq s \leq m}$ is a partition of $\Omega$.

b) $\left(\Omega_{r, i}\right)_{1 \leq r \leq i},\left(\Omega_{i+1, s}\right)_{i<s \leq m}$ and $\left(\Omega_{r, i} \cap \Omega_{i+1, s}\right)_{1 \leq r \leq i<s \leq m}$ are partitions of $\Omega \backslash \Omega_{i}$.

Proof. Is $x \notin \Omega_{i-1} \cup \Omega_{i}$ then $x \in \Omega_{i, i}$. If $x \in \Omega_{j}$, for $j \in\{i-1, i\}$ then $x \in \Omega_{r, s}$ where $r$ is the smaller integer such that $x \in \bigcap \Omega_{k=r}^{j}$ and $s$ is the biggest integer such that $x \in \bigcap \Omega_{k=j}^{s-1}$. And of course, if $(r, s) \neq\left(r^{\prime}, s^{\prime}\right)$ then $\Omega_{r, s} \cap \Omega_{r^{\prime}, s^{\prime}}=\emptyset$. The other proofs are identical.

The first alinea of the this lemma can be illustrated in the following figure, for $m=4$ and $i=2$ : in the left, we consider $\Omega$ and a general situation for $\Omega_{1}, \Omega_{2}$ and $\Omega_{3}$ and in the right, we have the sets $\Omega_{1,2}, \Omega_{1,3}, \Omega_{1,4}, \Omega_{2,3}$ and $\Omega_{2,4}$ that define a decomposition of $\Omega_{1} \cup \Omega_{2}$ (recall that $\Omega_{2,2}$ is $\Omega \backslash\left(\Omega_{1} \cup \Omega_{2}\right)$ ):
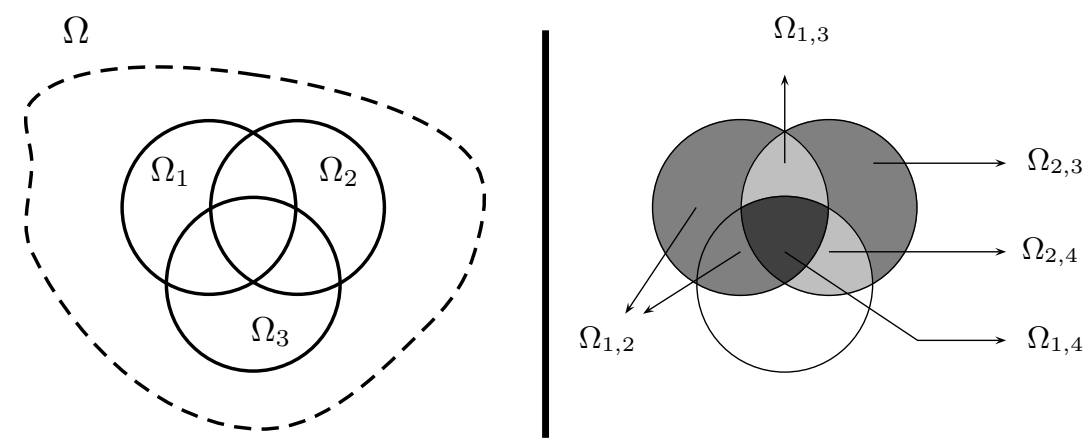

Figure 1. Decomposition of $\Omega_{1} \cup \Omega_{2}$, if $m=4$ and $i=2$. 


\section{Average function}

In what follows, given a set $A \subseteq \Omega, \chi_{A}$ denotes the characteristic function of $A$, i.e., $\chi_{A}(x)=1$ if $x \in A$ and $\chi_{A}(x)=0$ otherwise, and $\partial A$ the boundary of $A$. If $f_{1}, \ldots, f_{k} \in \mathcal{F}(\Omega)$ we denote their average $\left(\frac{f_{1}+\cdots+f_{k}}{k}\right)$ by $\left[f_{1}, \ldots, f_{k}\right]$. The average of elements of $\mathcal{F}(\Omega)^{m}$ are defined component by component.

Definition 2.1. With the notations above, we define the average function of $\mathcal{F}(\Omega)$ relatively to $\left(\Omega, \Omega_{1}, \ldots, \Omega_{m-1}\right)$ as $\psi=\left(\psi_{1}, \ldots, \psi_{m}\right): \mathcal{F}^{m}(\Omega) \rightarrow \mathcal{F}^{m}(\Omega)$ where

(2) $\forall i \leq m \quad \forall f=\left(f_{1}, \ldots, f_{m}\right) \in \mathcal{F}(\Omega)^{n} \quad \psi_{i}(f)=\sum_{r \leq i \leq s}\left[f_{r}, \cdots, f_{s}\right] \chi_{\Omega_{r, s}}$.

Note that, by Lemma 1.2 , for $1 \leq i \leq m$, and using the previous notations

$$
\forall r \leq i \leq s, \quad \psi_{i}(f)=\left[f_{r}, \cdots, f_{s}\right], \text { in } \Omega_{r, s} .
$$

and in particular

$$
\psi_{i}(f)=f_{i} \text {, in } \Omega \backslash\left(\Omega_{i-1} \cup \Omega_{i}\right) .
$$

If, for example, for some $k_{0} \leq m-1, \Omega_{k}$ is the empty set for all $k \leq k_{0}$ and is equal to $\Omega$, otherwise, then

$$
\psi_{i}(f)=\left\{\begin{array}{cl}
f_{i} & \text { if } i \leq k_{0} \\
{\left[f_{k_{0}+1}, \ldots, f_{m}\right]} & \text { if } i>k_{0}
\end{array}\right.
$$

In particular, if $k_{0}=0, \psi_{i}(f)=\left[f_{1}, \ldots, f_{m}\right]$ and, if $k_{0}=m-1, \psi$ is the identity function.

The average function have some nice (and trivial) properties. In particular it is linear and preserves averages.

Proposition 2.2. If $f, g, f^{1}, \ldots, f^{k} \in \mathcal{F}(\Omega)^{m}$ and $h \in \mathcal{F}(\Omega)$ then

a) $\psi(f+g)=\psi(f)+\psi(g)$;

b) $\psi(h f)=h \psi(f)$;

c) if $f \leq g$ then $\psi(f) \leq \psi(g)$;

d) $\psi\left(\left[f^{1}, \ldots, f^{k}\right]\right)=\left[\psi\left(f^{1}\right), \ldots, \psi\left(f^{k}\right)\right]$;

e) if $x \in \Omega$ and $f_{1}(x) \leq \cdots \leq f_{m}(x)$ then $\psi_{1}(f)(x) \leq \cdots \leq \psi_{m}(f)(x)$.

Proof. The first four alineas are immediate consequences of the definition of the average function. For the last alinea, suppose that $f_{1}(x) \leq \cdots \leq f_{m}(x)$ and let $1 \leq i<m$. If $x \in \Omega_{i}$ then $\psi_{i}(f)(x)=\psi_{i+1}(f)(x)$. If $x \notin \Omega_{i}$ then, by Lemma (1.2), $x \in \Omega_{r, i} \cap \Omega_{i+1, s}$ for some $r \leq i<s$ and, in this case $\psi_{i+1}(f)(x)=\left[f_{i+1}, \ldots, f_{s}\right](x) \geq$ $f_{i+1}(x) \geq f_{i}(x) \geq\left[f_{i}, \ldots, f_{r}\right](x)=\psi_{i}(f)(x)$. 
Remark 2.3. In fact the function $\psi$ should be denoted by $\psi^{\Omega, \Omega_{1}, \ldots, \Omega_{m-1}}$. We will use this notation only in the beginning of Section 3 and in the proof of the next result, where the use of an induction argument on $m$ may induce confusion.

As an immediate consequence of the definitions, we have, for $1 \leq j \leq i<m$

$$
\psi_{j}^{\Omega, \Omega_{1}, \ldots, \Omega_{m-1}}\left(f_{1}, \ldots, f_{m}\right)=\psi_{j}^{\Omega, \Omega_{1}, \ldots, \Omega_{i-1}}\left(f_{1}, \ldots, f_{i}\right) \quad \text { in } \Omega \backslash \Omega_{i} .
$$

Theorem 2.4. Given $m \geq 2$ and $\Omega_{1}, \ldots, \Omega_{m-1}$ subsets of $\Omega$, the average function $\psi=\left(\psi_{1}, \ldots, \psi_{m}\right)$ is the unique function satisfying the following conditions:

a) if $1 \leq i<m, \quad \psi_{i}\left(f_{1}, \ldots, f_{m}\right)=\psi_{i+1}\left(f_{1}, \ldots, f_{m}\right)$ in $\Omega_{i}$;

b) if $1 \leq i \leq m, \quad \sum_{j=1}^{i} \psi_{j}\left(f_{1}, \ldots, f_{m}\right)=\sum_{j=1}^{i} f_{j} \quad$ in $\Omega \backslash \Omega_{i}\left(\right.$ recall that, $\left.\Omega_{m}=\emptyset\right)$.

Proof. a) Note that $\psi_{i+1}\left(f_{1}, \ldots, f_{m}\right)-\psi_{i}\left(f_{1}, \ldots, f_{m}\right)$ is equal to

$$
\sum_{s=i+1}^{m}\left[f_{r}, \cdots, f_{s}\right] \chi_{\Omega_{i+1, s}}-\sum_{r=1}^{i}\left[f_{r}, \cdots, f_{s}\right] \chi_{\Omega_{r, i}}
$$

which vanish in $\Omega_{i}$ as $\Omega_{i+1, s} \cap \Omega_{i}=\Omega_{r, i} \cap \Omega_{i}=\emptyset$.

b) We will use an induction argument on $m$. If $i=1$ and $m \geq 2$ the result follows from (4). If $m=2$ (on the left) or $m \geq 3$ and $i=2$ (on the right) we have

$\left\{\begin{array}{l}\psi_{1}\left(f_{1}, f_{2}\right)=f_{1} \chi_{\Omega \backslash \Omega_{1}}+\frac{f_{1}+f_{2}}{2} \chi_{\Omega_{1}} \\ \psi_{2}\left(f_{1}, f_{2}\right)=f_{2} \chi_{\Omega \backslash \Omega_{1}}+\frac{f_{1}+f_{2}}{2} \chi_{\Omega_{1}}\end{array} \quad\left\{\begin{array}{l}\psi_{1}\left(f_{1}, \ldots, f_{m}\right)=f_{1} \chi_{\Omega \backslash \Omega_{1}}+\frac{f_{1}+f_{2}}{2} \chi_{\Omega_{1} \backslash \Omega_{2}} \\ \psi_{2}\left(f_{1}, \ldots, f_{m}\right)=f_{2} \chi_{\Omega \backslash \Omega_{1}}+\frac{f_{1}+f_{2}}{2} \chi_{\Omega_{1} \backslash \Omega_{2}}\end{array}\right.\right.$

For the induction step consider $m \geq 3$. If $2 \leq i<m$ then the results follows by the induction hypothesis using the equality (5). For the case $i=m$ we prove the result on each set $\Omega_{r, m}(r \leq m)$ that form a partition of $\Omega \backslash \Omega_{m}$ :

- in $\Omega_{m, m}=\Omega \backslash \Omega_{m-1}$ we have, using (5), with $i=m-1$, and the induction hypothesis,

$$
\begin{aligned}
\sum_{j=1}^{m} \psi_{j}\left(f_{1}, \ldots, f_{m}\right) & =\sum_{j=1}^{m-1} f_{j}+\psi_{m}\left(f_{1}, \ldots, f_{m}\right) \\
& =\sum_{j=1}^{m} f_{j}, \quad \text { by }(4)
\end{aligned}
$$

- in $\Omega_{1, m}=\Omega_{1} \cap \cdots \cap \Omega_{m-1}, \psi_{1}\left(f_{1}, \ldots, f_{m}\right)=\cdots=\psi_{m}\left(f_{1}, \ldots, f_{m}\right)=$ $\left[f_{1}, \ldots, f_{m}\right]$ by alinea a) and (3), and the result follows.

- in $\Omega_{r, m}=\Omega_{r} \cap \cdots \cap \Omega_{m-1} \backslash \Omega_{r-1}$ (for $1<r<m$ ) we have

$$
\sum_{j=1}^{r-1} \psi_{j}\left(f_{1}, \ldots, f_{m}\right)=\sum_{j=1}^{r-1} f_{j}
$$


using the case already proved. Then

$$
\begin{aligned}
\sum_{j=1}^{m} \psi_{j}\left(f_{1}, \ldots, f_{m}\right) & =\sum_{j=1}^{r-1} f_{j}+\sum_{j=r}^{m} \psi_{j}\left(f_{1}, \ldots, f_{m}\right) \\
& \left.=\sum_{j=1}^{r-1} f_{j}+(m-r+1) \psi_{m}\left(f_{1}, \ldots, f_{m}\right) \text { by alinea a }\right) \\
& =\sum_{j=1}^{m} f_{j} .
\end{aligned}
$$

as, by $(3)$, with $i=s=m, \psi_{m}\left(f_{1}, \ldots, f_{m}\right)=\left[f_{r}, \ldots, f_{m}\right]$.

To prove the uniqueness, suppose that $\varphi=\left(\varphi_{1}, \ldots, \varphi_{m}\right) \in \mathcal{F}(\Omega)^{m}$ satisfies conditions a) and b) and let us prove that $\varphi_{i}\left(f_{1}, \ldots, f_{m}\right)=\left[f_{r}, \ldots, f_{s}\right]$ in $\Omega_{r, s}$, for $1 \leq r \leq i \leq s \leq m$.

In fact, in $\Omega_{r, s}, \varphi_{r}\left(f_{1}, \ldots, f_{m}\right)=\cdots=\varphi_{s}\left(f_{1}, \ldots, f_{m}\right)$ and then

$$
\begin{aligned}
\varphi_{i}\left(f_{1}, \ldots, f_{m}\right) & =\frac{1}{s-r+1} \sum_{j=r}^{s} \varphi_{j}\left(f_{1}, \ldots, f_{m}\right) \\
& =\frac{1}{s-r+1}\left(\sum_{j=1}^{s} \varphi_{j}\left(f_{1}, \ldots, f_{m}\right)-\sum_{j=1}^{r-1} \varphi_{j}\left(f_{1}, \ldots, f_{m}\right)\right) \\
& =\frac{1}{s-r+1}\left(\sum_{j=1}^{s} f_{j}-\sum_{j=1}^{r-1} f_{j}\right) \quad \text { by condition b) } \\
& =\frac{1}{s-r+1} \sum_{j=r}^{s} f_{j}=\left[f_{r}, \ldots, f_{s}\right]
\end{aligned}
$$

which completes the proof.

We are now in conditions to studied when the average function of $f$ belongs to $\mathcal{B}^{m}$ and, if $\Omega$ is a topological space and $f$ is continuous, when this function is continuous.

Theorem 2.5. With the previous notations:

a) $\psi \circ \psi=\psi$;

b) $\mathcal{A}^{m}$ is the image of $\psi$;

c) $\mathcal{B}^{m}$ is the set of all $f=\left(f_{1}, \ldots, f_{m}\right) \in \mathcal{A}^{m}$ such that, if

$1 \leq r \leq i<s \leq m, x \in \Omega_{r, i} \cap \Omega_{i+1, s} \quad \Longrightarrow \quad\left[f_{r}, \ldots, f_{i}\right](x) \neq\left[f_{i+1}, \ldots, f_{s}\right](x)$.

d) if $\Omega$ is a topological space and $f=\left(f_{1}, \ldots, f_{m}\right)$ is a continuous functions of $\mathcal{A}^{m}$ then $\psi(f)$ is continuous if and only if

$1 \leq r, r^{\prime} \leq i<s, s^{\prime} \leq m, x \in \partial \Omega_{r, s} \cap \partial \Omega_{r^{\prime}, s^{\prime}} \quad \Longrightarrow \quad\left[f_{r}, \ldots, f_{s}\right](x)=\left[f_{r^{\prime}}, \ldots, f_{s^{\prime}}\right](x)$. 
Proof.

a) Let $j \in\{1, \ldots, m\}$. Then in $\Omega_{r, s}$, for $r \leq j \leq s$,

$$
\begin{aligned}
(\psi \circ \psi)_{j}\left(f_{1}, \ldots, f_{m}\right) & =\psi_{j}\left(\psi_{1}\left(f_{1}, \ldots, f_{m}\right), \ldots, \psi_{m}\left(f_{1}, \ldots, f_{m}\right)\right) \\
& =\left[\psi_{r}\left(f_{1}, \ldots, f_{m}\right), \ldots, \psi_{s}\left(f_{1}, \ldots, f_{m}\right)\right] \text { by }(2) \\
& =\left[\left[f_{r}, \ldots, f_{s}\right], \ldots,\left[f_{r}, \ldots, f_{s}\right]\right] \text { by }(2) \\
& =\left[f_{r}, \ldots, f_{s}\right]=\psi_{j}\left(f_{1}, \ldots, f_{m}\right) .
\end{aligned}
$$

b) By Theorem 2.4, the image of $\psi$ is contained in $\mathcal{A}^{m}$. For the other inclusion, just note that if $\left(f_{1}, \ldots, f_{m}\right) \in \mathcal{A}^{m}, 1 \leq r \leq i \leq s \leq m$, then $f_{r}=\cdots=f_{s}$ in $\Omega_{r, s}$ and by consequence $\psi_{i}\left(f_{1}, \ldots, f_{m}\right)=\left[f_{r}, \ldots, f_{s}\right]=f_{i}$.

c) Use the definition of $\psi$ and Lemma $1.2, \mathrm{~b})$.

d) Fix $i=1, \ldots, m$, and $f_{1}, \ldots, f_{m} \in \mathcal{F}(\Omega)$. We have a family of continuous functions $f_{r, s}: \Omega \rightarrow \mathbb{R}$ (with $1 \leq r \leq i \leq s \leq m$ ), defined by $f_{r, s}(x)=\left[f_{r}, \ldots, f_{s}\right]$, a partition of $\Omega,\left(\Omega_{r, s}\right)_{1 \leq r \leq i \leq s \leq m}$ and $\psi_{1}\left(f_{1}, \ldots, f_{m}\right): \Omega \rightarrow \mathbb{R}$ that is equal to $f_{r, s}$ in $\Omega_{r, s}$. In these conditions, it is well known that $\psi_{1}\left(f_{1}, \ldots, f_{m}\right)$ is continuous if and only if $f_{r, s}=f_{r^{\prime}, s^{\prime}}$ on $\partial \Omega_{r, s} \cap \partial \Omega_{r^{\prime}, s^{\prime}}$ for all $(r, s),\left(r^{\prime}, s^{\prime}\right)$.

Remark 2.6. If we use average functions with weight we obtain similar results. For instance if $\varepsilon_{1}, \ldots, \varepsilon_{m}>0$ and $\varepsilon_{1}+\cdots+\varepsilon_{m}=1$ then there exists a unique function $\Phi=\left(\Phi_{1}, \ldots, \Phi_{m}\right): \mathcal{F}^{m}(\Omega) \rightarrow \mathcal{F}^{m}(\Omega)$ such that for $\left(f_{1}, \ldots, f_{m}\right) \in \mathcal{F}^{m}(\Omega)$

a) if $1 \leq i<m, \quad \varepsilon_{i} \Phi_{i}\left(f_{1}, \ldots, f_{m}\right)=\varepsilon_{i+1} \Phi_{i+1}\left(f_{1}, \ldots, f_{m}\right)$ in $\Omega_{i}$;

b) if $1 \leq i \leq m, \quad \sum_{j=1}^{i} \varepsilon_{i} \Phi_{j}\left(f_{1}, \ldots, f_{m}\right)=\sum_{j=1}^{i} \varepsilon_{i} f_{j} \quad$ in $\Omega \backslash \Omega_{i}$.

The idea is to consider $\Phi_{i}\left(f_{1}, \ldots, f_{m}\right)=\frac{1}{\varepsilon_{i}} \psi\left(\varepsilon_{1} f_{1}, \ldots, \varepsilon_{m} f_{m}\right)$.

\section{The average function using characteristic functions}

Now we wish to define $\psi$ in terms of the characteristic functions of the coincidence sets $\bigcap_{j=k}^{n} \Omega_{j}$, with $1 \leq k, n \leq m$, with the convention that this intersection is equal to $\Omega$ if $k>n$. To simplify, we will denote those characteristic functions by $\chi_{k, n}$.

Using the equality $\chi_{A \backslash(B \cup C)}=\chi_{A}-\chi_{A \cap B}-\chi_{A \cap C}+\chi_{A \cap B \cap C}$, for $A, B, C \subseteq \Omega$, we obtain, for $i \leq m$ and $f_{1}, \ldots, f_{m} \in \mathcal{F}(\Omega)$,

(6) $\psi_{i}\left(f_{1}, \ldots, f_{m}\right)=\sum_{r \leq i \leq s}\left[f_{r}, \cdots, f_{s}\right]\left(\chi_{r, s-1}-\chi_{r-1, s-1}-\chi_{r, s}+\chi_{r-1, s}\right)$,

and the following result. 
Theorem 3.1. If $m \in \mathbb{N}, 1 \leq i \leq m$ and $f_{1}, \ldots, f_{m} \in \mathcal{F}$ then

$$
\psi_{i}\left(f_{1}, \ldots, f_{m}\right)=f_{i}+\sum_{1 \leq r<s \leq m, r \leq i \leq s} b(r, s, i) \chi_{r, s-1}
$$

where

$$
b(r, s, i)= \begin{cases}{\left[f_{r}, \ldots, f_{s}\right]-\left[f_{r}, \ldots, f_{s-1}\right]} & \text { if } i=r \\ {\left[f_{r}, \ldots, f_{s}\right]-\left[f_{r+1}, \ldots, f_{s}\right]} & \text { if } i=s \\ \frac{2}{(s-r)(s-r+1)}\left\{\left[f_{r+1}, \ldots, f_{s-1}\right]-\left[f_{r}, f_{s}\right]\right\} & \text { if } r<i<s .\end{cases}
$$

Proof. From equality (6) we obtain that $\psi_{i}\left(f_{1}, \ldots, f_{m}\right)$ is equal to

$$
\sum_{r \leq i \leq s}\left\{\left[f_{r}, \cdots, f_{s}\right]-\left[f_{r+1}, \cdots, f_{s}\right]-\left[f_{r}, \cdots, f_{s-1}\right]+\left[f_{r+1}, \cdots, f_{s-1}\right]\right\} \chi_{r, s-1}
$$

The only non trivial part is the one with $r<i<s$. In this case, if we multiply $\left[f_{r}, \cdots, f_{s}\right]-\left[f_{r+1}, \cdots, f_{s}\right]-\left[f_{r}, \cdots, f_{s-1}\right]+\left[f_{r+1}, \cdots, f_{s-1}\right]$ by $(s-r)(s-r+1)$ we obtain, successively

$$
\begin{array}{r}
(s-r) \sum_{k=r}^{s} f_{k}-(s-r+1)\left[\sum_{k=r+1}^{s} f_{k}+\sum_{k=r}^{s-1} f_{k}\right]+\frac{(s-r)(s-r+1)}{s-r-1} \sum_{k=r+1}^{s-1} f_{k} \\
-\left(f_{r}+f_{s}\right)+\left[(s-r)-2(s-r+1)+\frac{(s-r)(s-r+1)}{s-r-1}\right] \sum_{k=r+1}^{s-1} f_{k} \\
-\left(f_{r}+f_{s}\right)+\left[-(s-r+2)+\frac{(s-r)(s-r+1)}{s-r-1}\right] \sum_{k=r+1}^{s-1} f_{k} \\
-\left(f_{r}+f_{s}\right)+\frac{2}{s-r-1} \sum_{k=r+1}^{s-1} f_{k} \\
2\left(\left[f_{r+1}, \ldots, f_{s-1}\right]-\left[f_{r}, f_{s}\right]\right),
\end{array}
$$

which completes the proof.

Remark 3.2. The problem we considered in ([1]) was the following: given $\Omega$ a bounded open subset of $\mathbb{R}^{N}, E$ a Banach space contained in $\mathcal{F}(\Omega ; \mathbb{R})$, having some additional properties, a closed convex subset of $E^{N}$

$$
\mathbb{K}=\left\{\left(v_{1}, \ldots, v_{N}\right) \in E^{N}: v_{1} \geq \cdots \geq v_{N}\right\},
$$

$f_{1}, \ldots, f_{N} \in E^{\prime}$ and a given (not necessarily linear) operator $A: E \longrightarrow E^{\prime}$, we wanted to prove existence of $\left(u_{1}, \ldots, u_{N}\right) \in \mathbb{K}$ satisfying

$$
\sum_{j=1}^{N}\left\langle A u_{j}, v_{j}-u_{j}\right\rangle \geq \sum_{j=1}^{N}\left\langle f_{j}, v_{j}-u_{j}\right\rangle
$$

and to study some properties of this solution. With additional assumption on the $f_{j}, j=1, \ldots, N$, our aim was to prove that the inequality (8) could be rewritten as a 
system of equations, using the coincidence sets $\Omega_{i}=\left\{x \in \Omega: u_{i}(x)=u_{i+1}(x)\right\}$ and their characteristic functions. This characterization of the inequality by an equality had a decisive importance in the proof of the stability of these coincidence sets under small variations of the given functions $f_{j}, j=1, \ldots, N$.

\section{References}

[1] Azevedo, A. and Rodrigues, J. F. and Santos, L., The N-membranes problem for quasilinear degenerate systems, Interfaces and Free Boundaries, 7 (2005), 319-317. 